\title{
Direct Current Plasma Electron Source for Electric Propulsion Applications Using Atomic and Molecular Propellants
}

\author{
Antonio Gurciullo, Andrea Lucca Fabris and Aaron Knoll
}

\begin{abstract}
The design and performance of a novel direct current neutralizer for electric propulsion applications are presented. The neutralizer exploits an $\mathbf{E} \times \mathbf{B}$ discharge to enhance ionization via electron-neutral collisions. Tests are performed with helium, argon, xenon, air and water vapour as working gases. The $I$ $V$ characteristics and extraction parameters are measured for both atomic and molecular gases. The maximum partial power efficiency is $4.2 \mathrm{~mA} / \mathrm{W}$ in argon, $2.7 \mathrm{~mA} / \mathrm{W}$ in air and $2 \mathrm{~mA} / \mathrm{W}$ in water vapour. The typical utilization factor is below 1 and the power consumption is less than $120 \mathrm{~W}$. A semi-empirical model is derived to predict the performance of direct current plasma cathodes using atomic gas. A comparison with existing plasma cathodes and conventional $\mathrm{LaB}_{6}$ cathodes is presented and design optimisations aimed at improving the performance are proposed.
\end{abstract}

Index Terms-Electron sources, space vehicle propulsion, cathodes, magnetrons, plasma devices.

\section{INTRODUCTION}

$\mathbf{E}$ LECTRON sources are a fundamental component of several types of plasma thrusters, such as the Gridded Ion Engine (GIE) [1], the Hall Effect Thruster (HET) [2] and the Quad Confinement Thruster (QCT) [3]. Electron sources provide primary electrons in order to (i) initiate and sustain the plasma discharge in the thruster, and (ii) maintain charge conservation by balancing the current carried by the ejected ion beam. Since these electron sources neutralize the ion beam of a plasma thruster, they are also referred as neutralizers.

Conventional neutralizers exploit thermionic electron emission of a low work function insert material, such as barium oxide $(\mathrm{BaO})$ and lanthanum hexaboride $\left(\mathrm{LaB}_{6}\right)$. The insert material is typically heated to temperatures higher than $1000 \mathrm{~K}$ to trigger substantial emission current densities. $\mathrm{LaB}_{6}$ hollow cathodes with an emission current density of $20 \mathrm{~A} / \mathrm{cm}^{2}$ at approximately $2000 \mathrm{~K}$ have been reported [4]-[6]. These conventional neutralizers rely on the surface properties of the insert material and on the interactions of the insert with plasma and working gas.

Conventional neutralizers are affected by cathode poisoning. This is a chemical reaction between electronegative atoms

A. Gurciullo is a PhD Candidate at Surrey Space Centre, University of Surrey, Guildford, GU2 7XH, United Kingdom, e-mail: a.gurciullo@surrey.ac.uk.

A. Lucca Fabris is a Lecturer in Propulsion at Surrey Space Centre, University of Surrey, Guildford, GU2 7XH, United Kingdom, e-mail: a.luccafabris@surrey.ac.uk.

A. Knoll is a Senior Lecturer in Spacecraft Engineering at Imperial College London, Department of Aeronautics, South Kensington Campus, London, SW7 2AZ, United Kingdom, e-mail: a.knoll@imperial.ac.uk

Manuscript [info] or molecules and the insert material. The poisoning process causes an increase of the work function of the thermionic emitter. As a consequence, the electron emission is lowered or even quenched. Experimental poisoning characteristics of $\mathrm{LaB}_{6}$, which show the thermionic emission degradation as a function of the partial pressure of the poisoning gas and insert temperature, reveal the existence of a critical partial pressure of the poisoning gas [7], [8]. Above the critical partial pressure the thermionic emission of the insert decreases. Generally, this critical pressure rises with temperature of the insert material and depends on the electronegative property of the gas. For example, with $\mathrm{LaB}_{6}$ cathodes, oxygen has a critical pressure of $10^{-6}$ Torr at about $1700 \mathrm{~K}$ that rises to $10^{-4}$ Torr at about $1900 \mathrm{~K}[8]$.

A reduction of electron emission due to carbon contamination has also been observed [9], [10]. Oshima and Kawai [9] showed that at the temperature in which desorption of oxygen from $\mathrm{LaB}_{6}$ surface is enhanced, carbon poisoning is increased. The authors explained that carbon poisoning at elevated temperatures, above $1800 \mathrm{~K}$ in their tests, may be due to diffusion of carbon impurities from the bulk of the insert to its surface. At lower temperatures, below $1300 \mathrm{~K}$, carbon poisoning was due to adsorption of carbon on the insert surface, similar to the oxygen poisoning process.

One of the main research lines in the Electric Propulsion field is dedicated to identify possible alternative propellants (other than xenon) for EP systems. However, cathode poisoning may be a limiting factor for the many atomic and molecular propellants that could otherwise be considered for future EP systems. Alternative propellants may include molecular gases such as water vapour, air and compounds containing carbon. The main advantages of using alternative propellants are: (i) potential advantages in terms of cost, supply chain and availability; (ii) the same propellant can be used for on-board chemical and electrical propulsion systems; (iii) new mission scenarios can be enabled, such as air-breathing orbit and in-situ propellant harvesting on different planets; (iv) the system-level benefit of having the same propellant for electric and chemical propulsion can lead to cost, mass or volume savings on the spacecraft.

These two factors, insert poisoning of conventional neutralizers and growing interest in other propellants for plasma thrusters, motivate the design of novel neutralizers as alternatives to the hollow cathodes. Both $\mathrm{LaB}_{6}$ and barium oxide cathodes are high performing in terms of power efficiency and gas utilization if noble gases are used. However, the cathode 


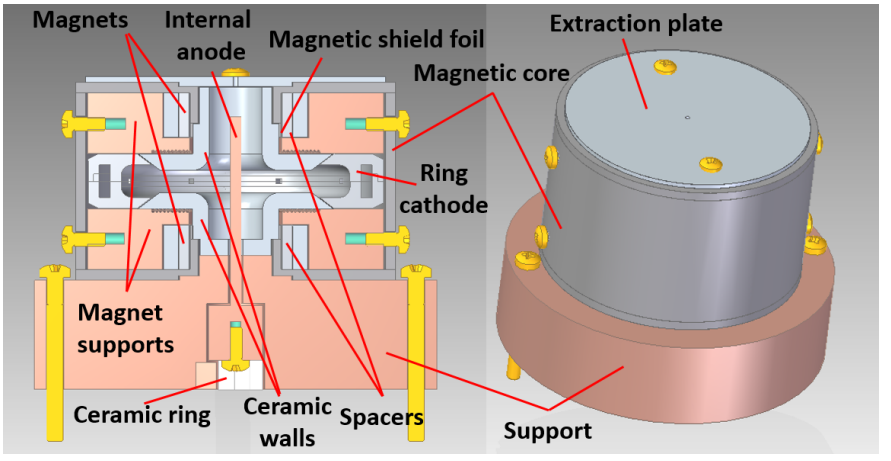

Fig. 1. Section and 3D view of the neutralizer design. (color online)

poisoning precludes their use with some of the alternative propellants.

Plasma cathodes have been considered as a potential replacement to conventional hollow cathodes. A plasma cathode does not rely on a thermionic emissive material and, instead, relies on ionization and electron generation in the bulk plasma [11]. Depending on the type of discharge, plasma cathodes can be grouped in radio frequency (RF) cathodes, capacitively coupled plasma (CCP) [12] and inductively couple plasma (ICP) [13], [14], microwave cathodes [15], [16], electron cyclotron resonance (ECR) cathodes [17], helicon cathodes [18], and direct current (DC) cathodes [19].

In this paper a novel direct current neutralizer which exploits an $\mathbf{E} \times \mathbf{B}$ region for ionization enhancement is presented. Since the main ionization mechanism is driven by a Hall current, the neutralizer was named Direct Current Hall Effect Neutralizer or DC-HallEN. This direct current plasma cathode has been designed and tested at the Surrey Space Centre, University of Surrey in Guildford, UK. The neutralizer was characterised using atomic propellants, such as $\mathrm{He}, \mathrm{Ar}, \mathrm{Kr}$ and $\mathrm{Xe}$, and molecular compounds, such as water vapour and air.

In this paper, experimental characterisation of the neutralizer is presented with and without electron extraction to an external anode. The results are shown for $\mathrm{He}, \mathrm{Ar}, \mathrm{Xe}$, air and water vapour. This paper is structured as follows. In Section II the design of the neutralizer, the experimental setup and the performance parameters are presented. The results of characterization of the neutralizer with and without electron extraction are provided in Section III. A semi-empirical performance model of a DC cathode working with atomic gases is introduced at the beginning of Section IV At the end of this section, the performance metrics of the DC-HallEN are compared with those of existing neutralizers.

\section{EXPERIMENTAL SETUP}

\section{A. The Direct Current Hall Effect Neutralizer (DC-HallEN)}

A cross section and a $3 \mathrm{D}$ view of the neutralizer design is shown in Fig. 1. The neutralizer is an axisymmetric device where the main components are a ring cathode, an internal anode, an extraction plate, two ceramic walls, two axially polarised ring magnets, a magnetic core, and various supports. The cathode is made of stainless steel and has a "C" cross section, where an internal cavity is used to diffuse the working

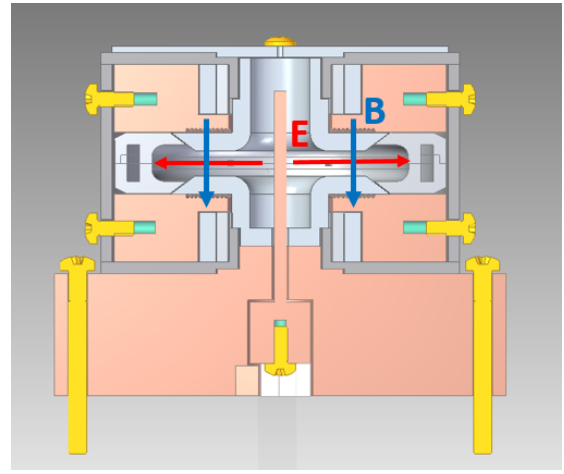

Fig. 2. $\mathbf{E} \times \mathbf{B}$ fields inside the neutralizer. (color online)

gas throughout the annular volume. This cavity is connected to a feed gas system through a $1 / 4$ in pipe. The gas is fed inside the discharge chamber through 8 equally spaced orifices bored in the inner surface of the ring cathode. The area of the cathode exposed to the plasma is about $18.8 \mathrm{~cm}^{2}$. The internal anode is a copper rod of $2 \mathrm{~mm}$ in diameter and extends axially about $44 \mathrm{~mm}$ inside the neutralizer. The extraction plate is made of aluminum. An extraction orifice is machined at the centre of this plate. Two ceramic walls made of boron nitride $(\mathrm{BN})$ are placed in the lower and the top side of the discharge chamber. The samarium cobalt ring magnets and the magnetic core form the magnetic circuit of the neutralizer. The support of the ring magnets and the support of the neutralizer are made of copper. The cathode and the internal anode are electrically insulated from the surrounding metal parts.

The neutralizer is designed to exploit a closed electron $\mathbf{E} \times$ $\mathrm{B}$ drift [20]. The drift velocity is defined by

$$
\mathbf{v}_{\mathbf{E} \times \mathbf{B}}=\frac{\mathbf{E} \times \mathbf{B}}{B^{2}}
$$

where $\mathbf{E}$ is the radial electric field and $\mathbf{B}$ is the quasi-axial magnetic flux density.

As shown in Fig. 2, the radial electric field and the axial magnetic field form a $\mathbf{E} \times \mathbf{B}$ region within the gap between the ceramic walls. As shown in Fig. 3 in this channel the peak magnetic flux density is slightly above $700 \mathrm{G}$. The ceramic channel and the magnetic flux density are dimensioned so that the following relations hold [21], [22]:

$$
\begin{gathered}
\lambda_{i z} \ll L \\
r_{L, e} \ll w \\
\omega_{c, e} \tau_{e} \gg 1
\end{gathered}
$$

where $\lambda_{i z}$ is the ionization mean free path, $L$ is the radial length of the ceramic channel, $r_{L, e}$ is the Larmor radius of electrons, $w$ is the axial thickness of the ceramic channel, $\omega_{c, e}$ is the gyro-frequency of electrons, and $\tau_{e}$ is the electronneutral collision period.

The strength of the magnetic field was tuned so that ions are unmagnetized while electrons are trapped in the $\mathbf{E} \times \mathbf{B}$ region before being collected by the internal anode or extracted from 


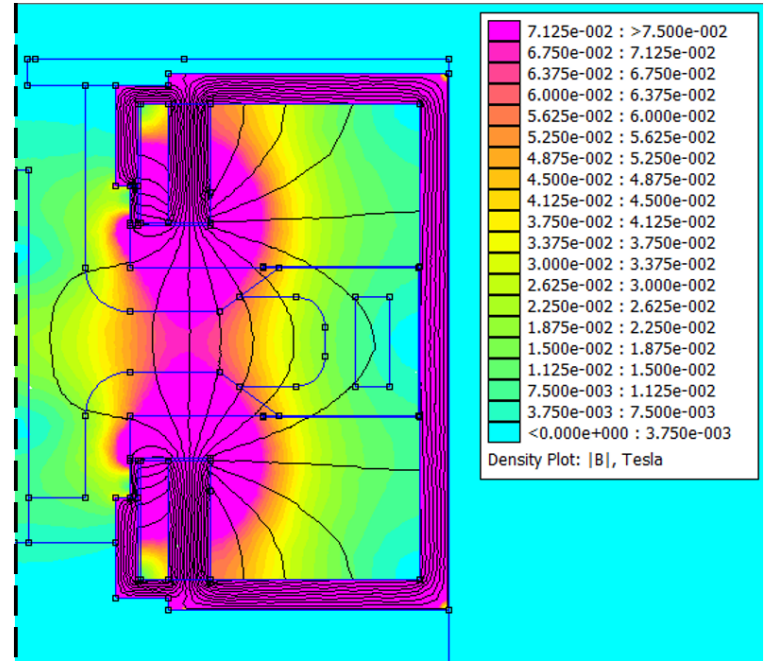

Fig. 3. Axisymmetric magnetic field inside the neutralizer simulated with FEMM [23]. (color online)

the neutralizer. The intensity of the magnetic field increases the residence time of the electrons inside the ceramic channel, thus, enhancing the probability of ionization events in that region. Along the axis of symmetry, the magnetic field has a null value, maintaining a low resistance path for electrons to the extraction orifice.

The extraction orifice is dimensioned according to the theory of electron extraction from a plasma source covered in [11] and [24]. From this theort it is anticipated that the most stable extraction would be achieved with an orifice of $1.8 \mathrm{~mm}$ diameter and a neutral pressure of 0.3 Torr inside the neutralizer.

\section{B. Experimental Equipment}

The experiments have been performed in Hermes vacuum chamber at the Surrey Electric Propulsion Laboratory. The pumping system, comprised of an oil diffusion pump, has a capacity of about $2000 \mathrm{l} / \mathrm{s}$ and throughput of about 4 Torr1/s. The laboratory equipment consists of two power supplies, a Glassman High Voltage Inc. model EQ50R24 (5000 V, $240 \mathrm{~mA}$ ) and a Sorensen DCS300- 4E (300 V, 4 A), a RS thermocouple reader model 615-8212, a Bronkhorst ELFLOW Select model F-201CV mass flow controller, a bespoke water vapour feed system, and, in some tests, a variable resistor $(0-100 \Omega)$ connected in series to the internal anode. Two thermocouples are used to measure the temperature of the annular cathode and the annular magnet close to the extraction plate.

For the tests with water vapour a low pressure gaseous feed system was designed. The feed system consists of a vessel made of glass for vacuum applications which contained liquid water, a pressure gauge to monitor the vapour pressure inside the vessel, and a temperature control unit to maintain the liquid water temperature constant during tests. The system exploits natural evaporation of water at low pressures and at a temperature slightly cooler than ambient. The vessel is connected to the neutralizer through a pipeline. The vessel and

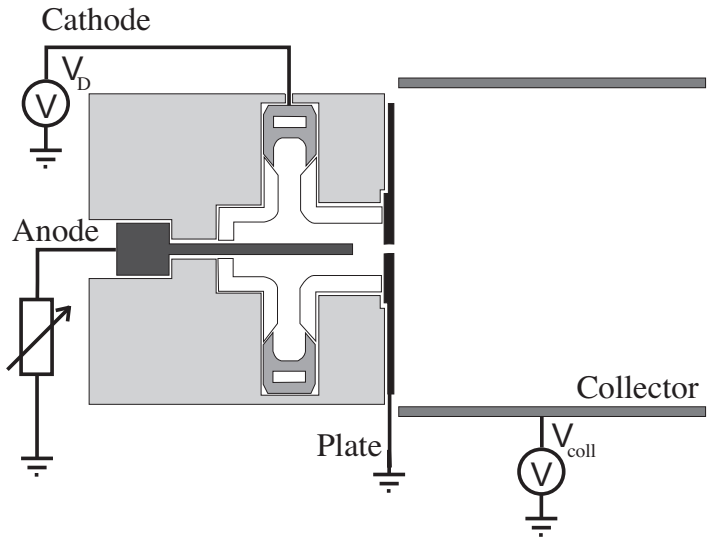

Fig. 4. Schematic of electrical connections.

the pressure gauge can be isolated from the vacuum chamber with valves. The temperature control unit consists of Peltier electro-thermal coolers and resistive heaters. The temperature is kept within the range $10-15{ }^{\circ} \mathrm{C}$, giving a vapour pressure of 9.2-12.8 Torr. The temperature is monitored with a K-type thermocouple attached to the liquid water vessel. The vessel is the coldest point of the entire system in order to avoid condensation along the pipeline leading to the cathode.

The water vapour mass flow rate is determined by measuring the volume of water evaporated through time at constant vessel temperature and pressure. For the results presented in this paper, the estimated water vapour mass flow rate is $0.22 \pm 0.02$ $\mathrm{mg} / \mathrm{s}$ (approximately 16.5 SCCM).

The neutralizer is supported by a copper pillar with an embedded water cooling system. The external collector consists of a copper hollow cylinder of internal diameter of $7.6 \mathrm{~cm}$ and axial length of $10 \mathrm{~cm}$. The collector is placed coaxially in front of the neutralizer. This layout avoids backscattering of neutrals exiting the neutralizer.

A schematic of the electrical connections is shown in Fig. 4 The cathode is connected to the negative output of a power supply $(5000 \mathrm{~V}, 240 \mathrm{~mA})$ which is controlled in current limited mode during tests. The collector is connected to a second power supply ( $300 \mathrm{~V}, 4 \mathrm{~A})$ and positively biased relative to the ground. The anode and the extraction plate are connected to the chamber walls, which is the electrical ground of the power supplies. The extracted current is measured by a multimeter placed in series between the collector and the second power supply. The current and voltage of the cathode are reported based on the display reading of the $5000 \mathrm{~V}$ power supply with a voltage resolution of $10 \mathrm{~V}$.

In order to operate the neutralizer, the gas is introduced through the cathode. The cathode voltage is incrementally set at high negative values until a breakdown event occurs. Ions are collected at the ring cathode while some electrons are trapped in the $\mathbf{E} \times \mathbf{B}$ region. Within the channel volume, further electron-neutral collisions enhance the probability of ionization events. Then, most of the electrons are either collected on the internal anode or extracted through the extraction orifice, reaching the external collector, while a small number of electrons are likely lost on the extraction plate. 


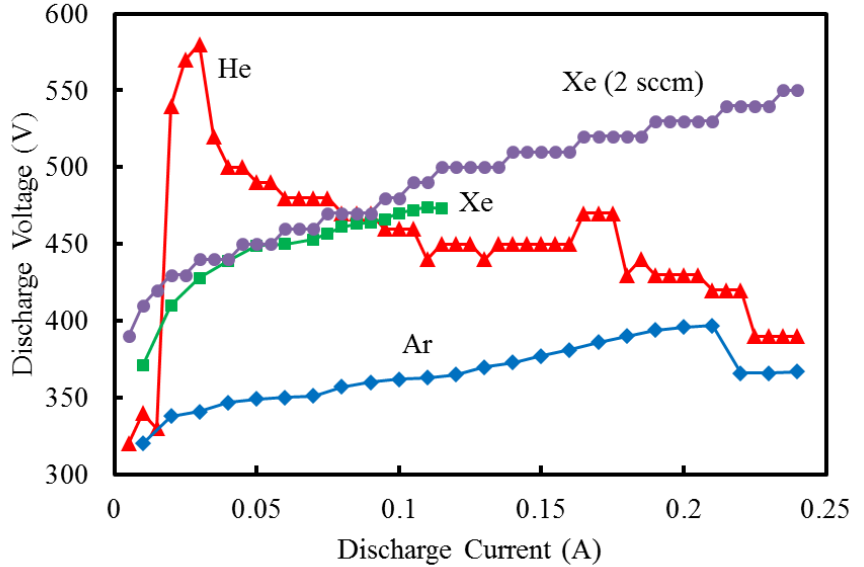

(a)

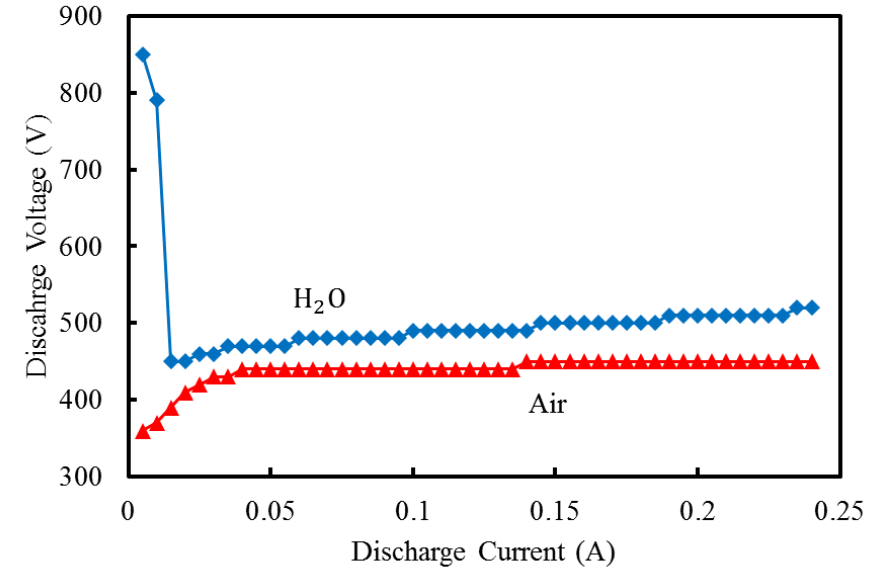

(b)

Fig. 5. $I-V$ characteristics of the neutralizer without electron extraction for (a) atomic and (b) molecular gases. The atomic gases are He, Ar and Xe at flow rate of 7.5 SCCM and a second curve for Xe at a flow rate of 2 SCCM. The molecular gases are air at 3.5 SCCM and $\mathrm{H}_{2} \mathrm{O}$ at 16.5 SCCM. (color online)

\section{Performance Parameters}

The neutralizer is tested in two conditions: without and with electron extraction. At the condition without extraction, the external collector is not powered, electrons are collected on the internal anode and the $I-V$ characteristic of the neutralizer is recorded. At the condition with extraction, the external collector is powered in voltage limited mode and the electrons are mainly collected on the external anode or the extraction plate. During extraction tests, four performance parameters have been calculated: the extraction parameter $\alpha$, the partial power efficiency $H_{p}$, the total power efficiency $H_{t}$ and the gas utilization factor $U$. These parameters are defined as follows:

$$
\begin{gathered}
\alpha=\frac{I_{\text {extr }}}{I_{D}} \\
H_{p}=\frac{I_{\text {extr }}}{P_{D}} \\
H_{t}=\frac{I_{\text {extr }}}{P_{D}+P_{\text {extr }}} \\
U=\frac{I_{\text {extr }}}{C \dot{m}_{g}}
\end{gathered}
$$

where $I_{D}$ is discharge current, $I_{\text {extr }}$ is extracted electron current, $P_{D}=I_{D} V_{D}$ is the discharge power, $V_{D}$ is the discharge voltage, $P_{\text {extr }}$ is the power measured at the external collector, $\dot{m}_{g}$ is the gas flow rate in standard cubic centimetres per minute (SCCM), and $C=0.07174 \mathrm{~A} / \mathrm{SCCM}$ is a constant. This constant is the equivalent current per SCCM of atomic gas that can be produced assumed all atoms are subjected to single ionization [25]. The gas utilization factor represents the number of ionization-neutralization cycles a neutral particle undergoes before escaping the neutralizer.

It is important to note that the extraction power $P_{\text {extr }}$ depends on the geometry of the collector and on the distance between the neutralizer and the collector. Therefore, the use of the partial power efficiency $H_{p}$ is more appropriate than the total one for comparing the performance of various neutralizes, since it depends only on the discharge condition inside the device.

\section{RESULTS}

The DC-HallEN has been characterised with $\mathrm{He}, \mathrm{Ar}, \mathrm{Xe}$, air and water vapour. Three atomic gases have been chosen to study the influence of mass and ionization energy of an atom on the neutralizer performances.

The $I-V$ characteristics of the neutralizer measured without electron extraction are shown in Fig. 5. These characteristics have been recorded with both atomic and molecular gases.

Fig. 5a illustrates curves for $\mathrm{He}, \mathrm{Ar}$ and Xe. The flow rate is $7.5 \mathrm{SCCM}$ except for a second curve of Xe in which the flow rate was 2 SCCM. Generally, $I-V$ curves of Ar and Xe present a positive slope, and the discharge voltage is higher when using Xe. This feature is due to the balancing of three factors: (i) ionisation and excitation cross-sections, (ii) ion mass and (iii) secondary electron emission which depends on the gas-surface material interaction. An increase of the flow rate induces a shift of the $I-V$ toward lower discharge voltages, as is shown by the comparison of the two curves of xenon. A typical $I-V$ curve of He has a peak at very low current and a negative slope within the discharge current range 0.04-0.24 A. The abrupt increase of the discharge voltage at very low currents may be due to a transition from a localized ionization in the vicinity of the cathode to a full discharge along the space between the cathode and the anode. After the peak, the voltage decreases with the current. This behaviour is opposite to that one observed with Ar and Xe and may be due to a strong influence of the magnetic field on He ions motion. The radial thickness of the strong magnetic field region $(700 \mathrm{G})$ is below $3 \mathrm{~mm}$. In this region, assuming a thermal energy of newborn ions of $T_{i}=0.1 \mathrm{eV}$, the Larmor radius of xenon ion is about $8.4 \mathrm{~mm}$. At the same conditions, the Larmor radius of $\mathrm{He}$ ions is about 3.2 and 5.7 times smaller than $\mathrm{Ar}$ and Xe ions, respectively. Therefore, it is thought that the magnetic field has a strong influence on He ions, which may 


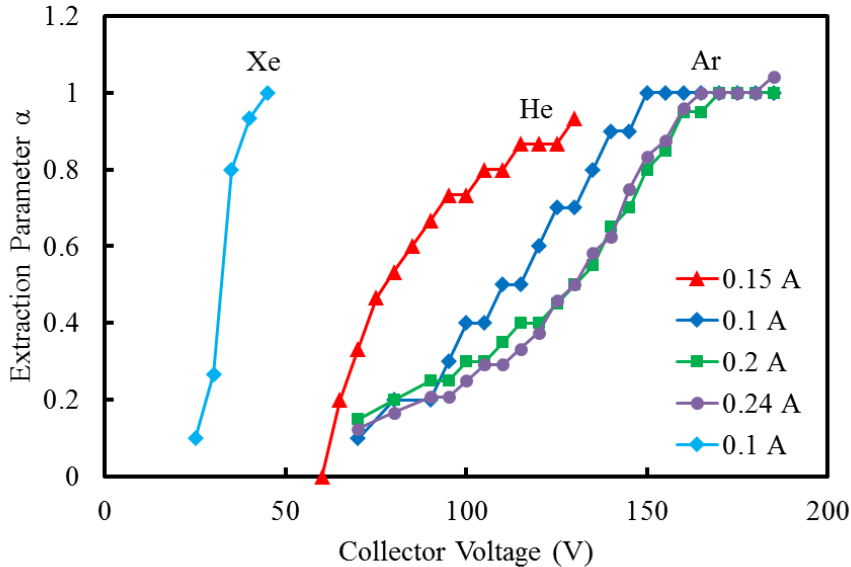

(a)

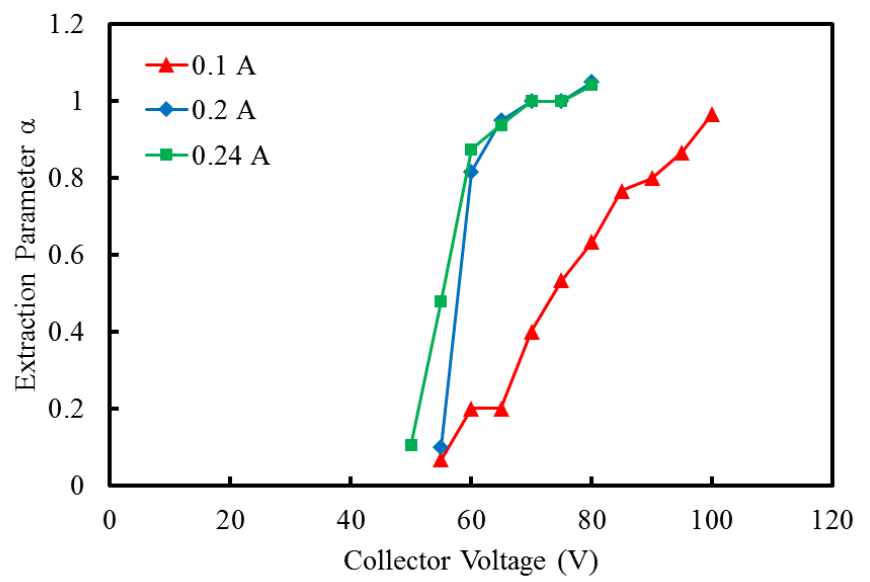

(c)

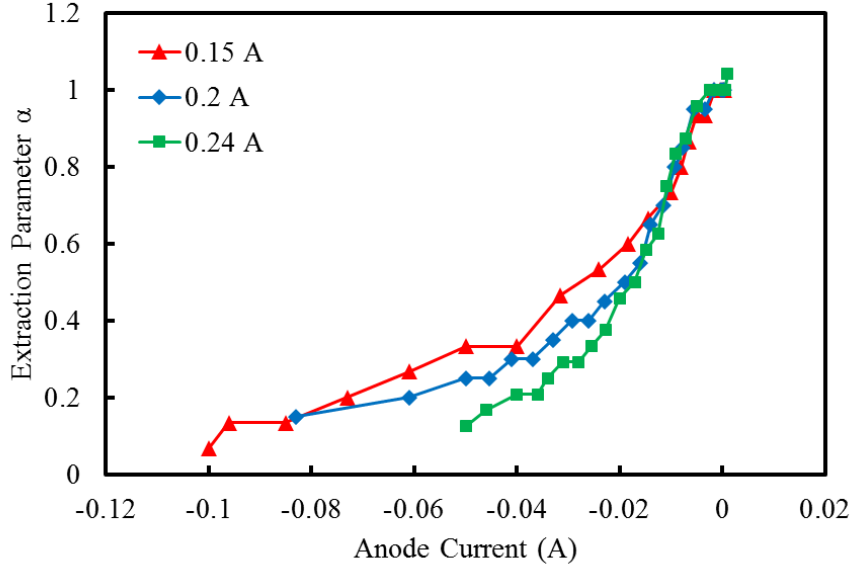

(b)

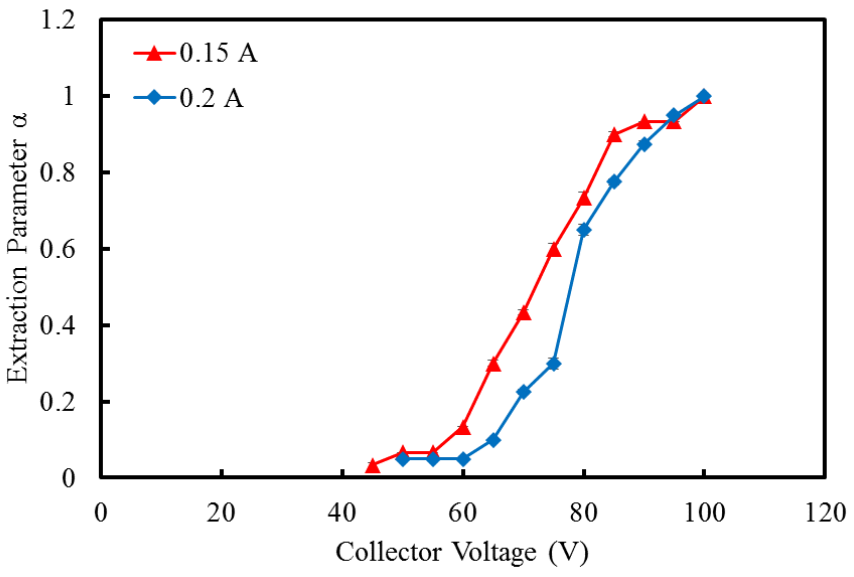

(d)

Fig. 6. Extraction parameter $\alpha$ versus collector voltage for (a) He, Ar and Xe. The flow rates are 5 SCCM for He and Ar, and 2 SCCM for Xe. (b) Extraction parameter $\alpha$ versus internal anode current for Ar at a flow rate of 5 SCCM. Extraction parameter $\alpha$ versus collector voltage for (c) air at 3.5 SCCM and (d) $\mathrm{H}_{2} \mathrm{O}$ at 16.5 SCCM. The curves are for various discharge currents. (color online)

be temporarily trapped in the radial channel and prevented to reach the cathode along straight paths.

The $I-V$ characteristics of air and water vapour are shown in Fig. 5b. The flow rates of air and water vapour are 3.5 SCCM and 16.5 SCCM, respectively. Both curves have positive slopes within the current range of $0.02-0.24 \mathrm{~A}$. The experimental profile obtained with water vapour presents a peak at low discharge current and, in general, is characterised by a higher discharge voltage in comparison with air. Moreover, the slope of the $\mathrm{H}_{2} \mathrm{O} I-V$ characteristic is steeper than for air.

A second set of experiments have been performed implementing external electron extraction. The curves of the extraction parameter $\alpha$ versus the collector voltage for $\mathrm{He}$, $\mathrm{Ar}$ and Xe are shown in Fig. 6a The data were collected with a gas flow rate of 5 SCCM and at various discharge currents. All the curves exhibit a decrease in slope, suggesting that a saturation may occur at higher voltage values. At a fixed diameter of the extraction orifice the collector voltage at which the saturation takes place depends on the type of gas, discharge current and gas flow rate. With xenon, full extraction is achieved at a lower collector voltage than for the other atomic gases. The dependence of the $\alpha-V$ curve on the discharge current is illustrated by a set of three experimental profiles for argon. The $\alpha-V$ curve shifts to higher voltage as the discharge current is increased. Moreover, the profiles at higher currents tend to overlap.

The curves of the extraction parameter $\alpha$ versus the collector voltage for air and water vapour are shown in Fig. 6c and Fig. $6 \mathrm{~d}$. The flow rate of air and water vapour are $3.5 \mathrm{SCCM}$ and 16.5 SCCM, respectively. The dependence of the extraction parameter on the discharge current is shown. Generally, using air, an increase of the discharge current corresponds to an increase of the extraction parameter and the electron extraction reaches saturation within a smaller collector voltage range than in the case of water vapour.

As we can observe in Fig. 6a, Fig. 6c and Fig. 6d, an extraction parameter higher than 1 is also recorded. In order to investigate this, the current flowing through the internal anode is compared to the extraction parameter, as shown in Fig. 6b. Negative currents correspond to a flow of electrons collected by the internal anode from the plasma. Positive currents correspond to the opposite, where positively charged particle are collected by the internal anode or negatively charged particles are emitted by the internal anode. When the 


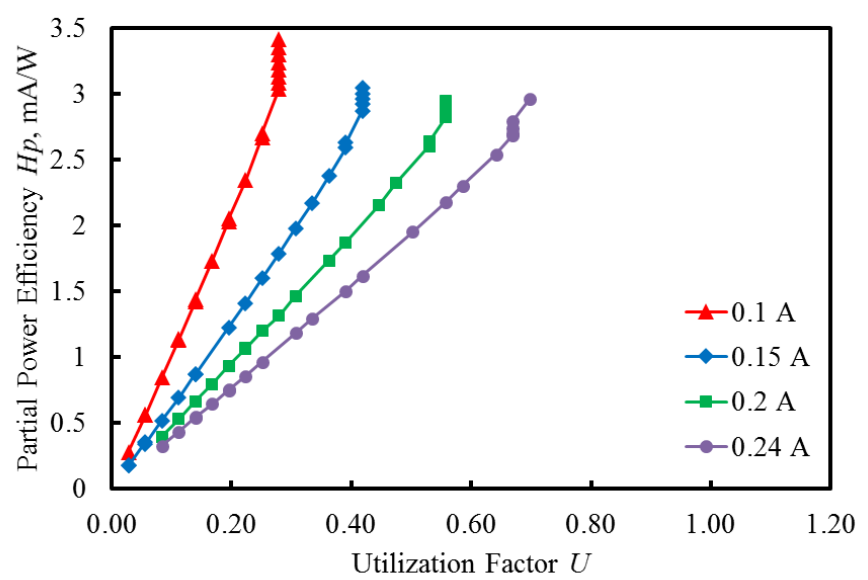

(a)

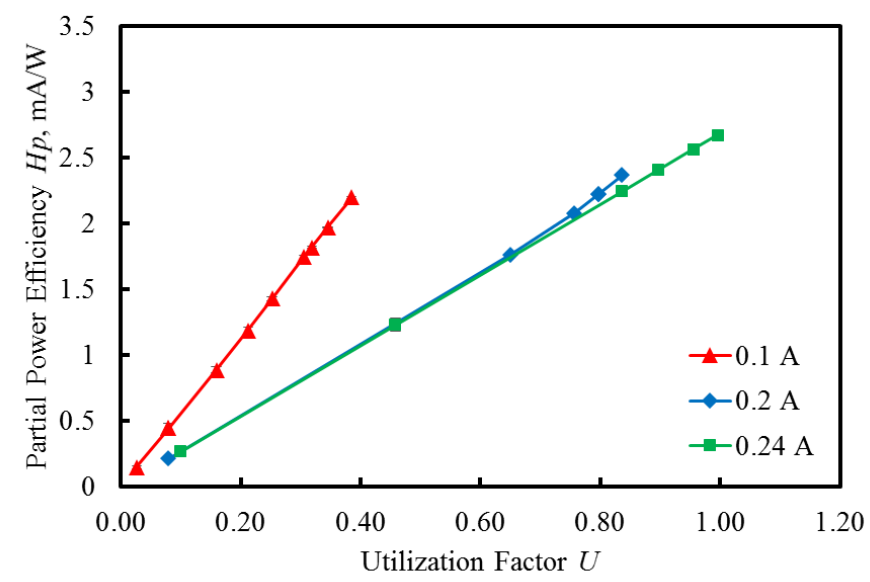

(b)

Fig. 7. Partial power efficiency versus utilization factor in (a) Ar at 5 SCCM and (b) air at 3.5 SCCM. The curves are for various discharge currents. (color online)

extraction parameter approaches 1 , the current collected by the internal anode is null. Moreover, if the extraction parameter is higher than 1 , the internal anode current is positive. Since $\alpha=I_{\text {extr }} / I_{D}$, the surplus of electron current collected by the external collector originates from ionization events taking place between the internal anode and the collector, or even outside the neutralizer, between the extraction plate and the collector.

In Fig. 7 the relation between the partial power efficiency $H_{p}$ and the gas utilization factor $U$ in argon and air is shown. Within a certain range, $H_{p}$ linearly increases with $U$. As it will be shown in the discussion section, for atomic gases two extraction regions can be identified by examining the $H_{p}-U$ plots.

For all atomic gases tested, the $H_{p}-U$ plots have a pattern as the one shown in Fig. 7, i.e. they have a region with a constant positive slope and a region where $H_{p}$ increases at a fixed maximum utilization factor. The definition of gas utilization factor in (8) is valid for atomic gases, since it takes into account only single ionization of a monoatomic species. $U$, as it is defined, loses its physical meaning when applied to molecular gases, where dissociation and ionization of multiple species occur contributing to the overall equivalent current. However, this parameter may be used as a guideline to compare ionization efficiency of molecules relative to atomic gases by assuming ionization of the molecule without any dissociation events. A $H_{p}-U$ plot for air is shown in Fig. 7b A similar plot for water vapour was not possible to record because the discharge voltage during extraction was not stable.

Some of the performance parameters of the DC-HallEN recorded during the experimental campaign using atomic and molecular gases are listed in Table. I Most of the investigations where performed with $\mathrm{Ar}$ as a working gas, where a broad range of discharge parameters were examined. Generally, the maximum partial power efficiency is $2-4 \mathrm{~mA} / \mathrm{W}$, the gas utilization factor below 1 and a power needed to operate the neutralizer up to $120 \mathrm{~W}$.

Since water is a compound more complex than air, the energy cost per single ionization event may be higher for water. Electron collisions with molecules cause dissociation, vibrational and rotational excitations, scattering and ionization. Thus, in molecular gases it is expected a higher energy cost per single ionization event. At the same time, the power cost depends on collisional cross-sections, ion mass and secondary electron emission of the cathode material.

\section{Discussion}

\section{A. Theory on Performance of a Direct Current Plasma Neu- tralizer Using Atomic Propellant}

The partial power efficiency in (6) and the gas utilization factor in (8) can be written as functions of the extraction parameter $\alpha$ :

$$
\begin{gathered}
H_{p}=\frac{\alpha}{V_{D}} \\
U=\frac{\alpha I_{D}}{C \dot{m}_{g}} .
\end{gathered}
$$

TABLE I

Performance Parameters of THE DC-HALlEN for ATOMic AND MOlecular GaSes

\begin{tabular}{lcccccc}
\hline \hline & $\mathrm{He}$ & $\mathrm{Ar}$ & $\mathrm{Kr}$ & $\mathrm{Xe}$ & air & $\mathrm{H}_{2} \mathrm{O}$ \\
\hline $\begin{array}{l}\text { Maximum partial power } \\
\text { efficiency } H_{p}[\mathrm{~mA} / \mathrm{W}]\end{array}$ & 2.1 & 4.2 & 2.6 & 1.9 & 2.7 & 2.0 \\
$\begin{array}{l}\text { Maximum utilization } \\
\text { factor } U\end{array}$ & 0.4 & 0.7 & 0.7 & 0.7 & 1.1 & 0.1 \\
Discharge power [W] & up to 70 & $30-90$ & $40-110$ & up to 50 & $45-90$ & up to 120 \\
\hline \hline
\end{tabular}




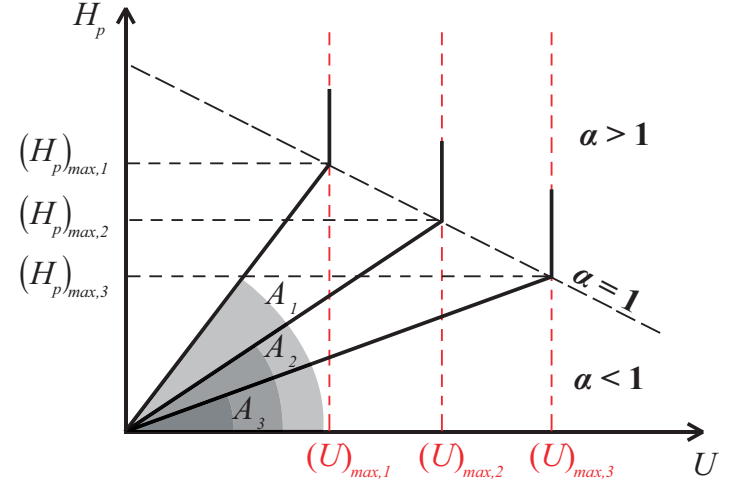

(a)

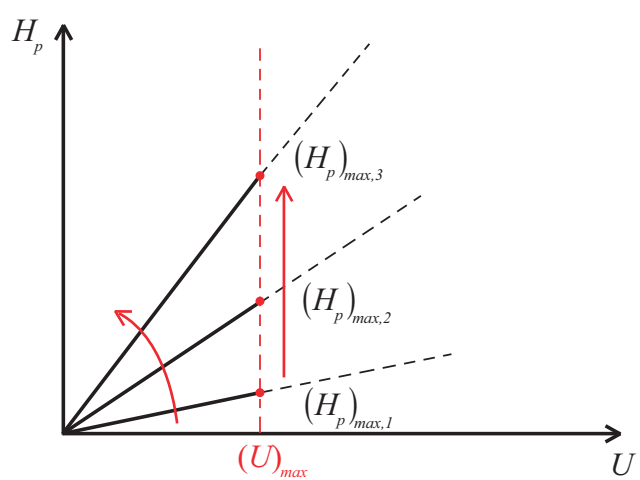

(b)

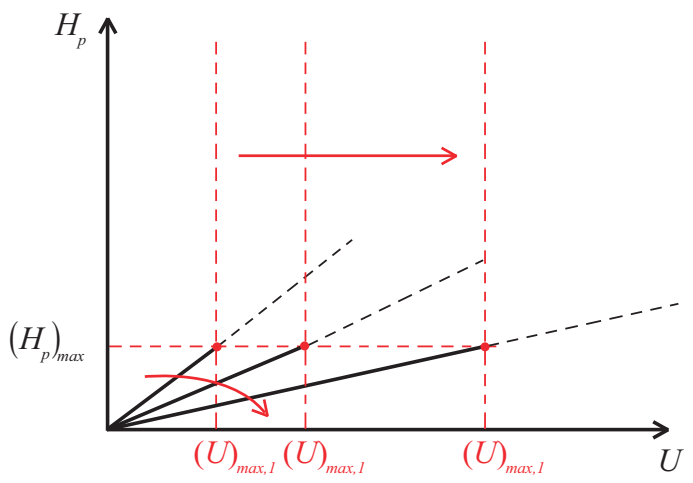

(c)

Fig. 8. (a) Features of a $H_{p}-U$ characteristic. Influence of (b) the discharge voltage, (c) discharge current and gas flow rate on the $H_{p}-U$ characteristic. (color online)

As shown in Fig. 7a, the $H_{p}-U$ curve is approximately linear within a certain range and the following relation exhists:

$$
H_{p}=A U
$$

where the slope of this linear function is

$$
A=\frac{C \dot{m}_{g}}{I_{D} V_{D}}
$$

The trend of the $H_{p}-U$ curve can be explained as follows. Generally, the discharge voltage decreases while the extracted current increases. At $\alpha<1$, the discharge voltage $V_{D}$ decreases at a rate much lower than the increase rate of the extraction current. In this region, the $A$ parameter defined in (12) is approximately constant. At $\alpha=1$, the electron current extracted from the neutralizer reaches its maximum. At this point the discharge voltage is slightly lower than at $\alpha=0$ and it was observed that the rates of change of $V_{D}$ and $I_{\text {extr }}$ reverse, causing the formation of a knee in the plots. At $\alpha>1$ the extraction current increases at a lower rate than the decrease rate of the discharge voltage. In this second region, some of the electrons created in the gap between the internal anode and the external collector contribute to the extraction current. The regions of the $H_{p}-U$ curves are schematically showed in Fig. 8a.

Due to the dependences of $H_{p}$ and $U$ on the discharge voltage, discharge current and gas flow rate, it is possible to predict the performance of a DC neutralizer that uses atomic gases. Three main cases can be identified:
Case (i). If the discharge voltage $V_{D}$ decreases, it follows that

$$
\left(H_{p}\right)_{\max } \sim \frac{1}{V_{D}}, \text { increases }
$$

$$
\begin{aligned}
& A \sim \frac{1}{V_{D}}, \text { increases } \\
& (U)_{\max }=\text { constant }
\end{aligned}
$$

Case (ii). If the discharge current $I_{D}$ increases, it follows that

$$
\begin{gathered}
\left(H_{p}\right)_{\max }=\text { constant } \\
A \sim \frac{1}{I_{D}}, \text { decreases } \\
(U)_{\max } \sim I_{D}, \text { increases }
\end{gathered}
$$

Case (iii). If the gas flow rate $\dot{m}_{g}$ decreases, it follows that

$$
\begin{gathered}
\left(H_{p}\right)_{\max }=\text { constant } \\
A \sim \dot{m}_{g}, \text { decreases } \\
(U)_{\max } \sim \frac{1}{\dot{m}_{g}}, \text { increases }
\end{gathered}
$$


TABLE II

TYPICAL PERFORMANCES OF NEUTRALIZERS FOR SPACE PROPULSION APPLICATIONS

\begin{tabular}{|c|c|c|c|c|c|c|}
\hline$\#$ & $\begin{array}{l}\text { Neutralizer Type, } \\
\text { working gas }\end{array}$ & $\begin{array}{l}\text { Gas flow } \\
\text { rate }[\mathrm{SCCM}]\end{array}$ & $\begin{array}{l}\text { Extracted electron } \\
\text { current }[\mathrm{A}]\end{array}$ & $\begin{array}{c}\text { Input } \\
\text { power [W] }\end{array}$ & $\begin{array}{c}\text { Partial power } \\
\text { efficiency } H_{p}[\mathrm{~mA} / \mathrm{W}]\end{array}$ & $\begin{array}{l}\text { Gas utilization } \\
\text { factor } U\end{array}$ \\
\hline 1 & DC-HallEN, argon & 10.0 & 0.24 & 68 & 3.5 & 0.3 \\
\hline 2 & CCP, xenon $[12]$ & 0.5 & 0.12 & 40 & 2.9 & 3.2 \\
\hline 3 & ICP, xenon $[26]$ & 3.0 & 3.30 & 140 & 23.6 & 15.3 \\
\hline 4 & ICP, argon |13], |27| & 3.0 & 0.40 & 50 & 8.0 & 1.9 \\
\hline 5 & Microwave, xenon [28] & 7.5 & 2.10 & 60 & 35.0 & 3.9 \\
\hline 6 & ECR, xenon [17] & 3.5 & 2.35 & 125 & 18.8 & 9.4 \\
\hline 7 & ECR, argon [29] & 5.0 & 2.45 & 600 & 4.1 & 6.8 \\
\hline 8 & Helicon, xenon |18, |30], |31] & 2.2 & 30.00 & 1300 & 23.1 & 190.1 \\
\hline 9 & Helicon, argon $|18|,|30|, \mid 31]$ & 15.0 & 15.00 & 1200 & 12.5 & 13.9 \\
\hline 10 & $\mathrm{LaB}_{6}$, xenon $[5$ & 10.0 & 60.00 & 1260 & 47.6 & 83.6 \\
\hline
\end{tabular}

Case (i) is shown in Fig. $8 \mathrm{~b}$, cases (ii) and (iii) in Fig. $8 \mathrm{c}$

\section{B. Comparison With Other Plasma Cathodes Using Noble Gases}

A list of neutralizers for electric propulsion applications and respective performances with atomic gases are listed in Table. III. It is possible to note that the novel neutralizer proposed in this paper is lower performing when compared to most of the other plasma cathodes and $\mathrm{LaB}_{6}$ cathodes. At this stage, the DC-HallEN has a partial power efficiency one order of magnitude lower than $\mathrm{LaB}_{6}$ cathode. However, if it is compared to plasma cathodes operating at a similar power range, such as the ones in line 2 and 4, the partial power efficiency may be in the same order of magnitude. The gas utilization factor of the DC-HallEN is much lower than the other neutralizers. These results suggest that an optimisation of the ionization mechanism is required to improve the performance of this novel neutralizer concept. However, the DC-HallEN relies on electron production in volume rather than from surface. This enables the neutralizer to operate with a wide range of propellants.

\section{CONCLUSION}

The design and performance of a novel direct current neutralizer for electric propulsion application have been presented. The neutralizer consists of an axisymmetric plasma cathode where a ring cathode surrounds an axial internal anode. A set of permanent magnets produces a quasi-axial magnetic field having peak value of slightly above $700 \mathrm{G}$. The main ionization mechanism of the neutralizer is a discharge in $\mathbf{E} \times \mathbf{B}$ fields. A closed loop current drift, known as Hall current, increases the residence time of electrons inside a radial ceramic channel, enhancing the probability of ionization events in that region. Due to this ionization mechanism and mode of operation, the neutralizer is named Direct Current Hall Effect Neutralizer (DC-HallEN).

Tests were performed using atomic gases, such as $\mathrm{He}, \mathrm{Ar}$ and $\mathrm{Xe}$, and molecular gases, such as air and water vapour. The tests were conducted with and without electron extraction. The $I-V$ characteristics of the DC-HallEN showed that the discharge voltage increases with the discharge current except when using He. The discharge voltage of water vapour was higher than air, likely due to a higher energy cost per ionization when using water vapour.

The ratio of the extracted electron current versus the discharge current was plotted against the voltage of an external collector. At high collector voltages, electrons created during ionizations events between the internal anode and the external collector contributed to the total extracted electron current.

The current flowing through the internal anode was investigated during electron extraction. It was shown that no current flowed through it when the extracted electron current matched with the discharge current.

A semi-empirical model for predicting the performance of a direct current plasma neutralizer using atomic gases was presented. The model gives a predictive relationship between the partial power efficiency $H_{p}$ and the gas utilization factor $U$. The influence of gas flow rate, discharge voltage and discharge current on a $H_{p}-U$ characteristic was explained. using this simple model.

The results confirmed that an optimization of the electron production mechanism should be pursued in order to increase the extraction performances of the DC-HallEN. With air, the maximum partial power efficiency and the maximum utilization factor were $2.7 \mathrm{~mA} / \mathrm{W}$ and 1.1 , respectively, when a discharge power of 45-90 W was applied. With water vapour, the maximum partial power efficiency and the maximum utilization factor were $2 \mathrm{~mA} / \mathrm{W}$ and 0.1 , respectively, when a discharge power up to $120 \mathrm{~W}$ was applied. Among the atomic gases, the performance with argon were the most investigated. With this gas, the maximum partial power efficiency and the maximum utilization factor were $4.2 \mathrm{~mA} / \mathrm{W}$ and 0.7 , respectively, when a discharge power of 30-90 W was applied.

\section{ACKNOWLEDGMENT}

This work is part of the strategic partnership between University of Surrey and Airbus Defence and Space. The authors would also like to thank Surrey Satellite Technology Ltd for sponsoring the $\mathrm{PhD}$ studentship that enabled this research to be conducted.

\section{REFERENCES}

[1] J. R. Anderson, K. D. Goodfellow, J. E. Polk, V. K. Rawlin, and J. S. Sovey, "Performance characteristics of the NSTAR ion thruster during an on-going long duration ground test," IEEE Aerospace Conference Proceedings, vol. 4, no. January, pp. 99-121, 2000. 
[2] J. R. Brophy, "Stationary Plasma Thruster Evaluation in Russia," tech. rep., Jet Propulsion Laboratory, Pasadena, 1992.

[3] A. Knoll, D. Lamprou, V. Lappas, M. Pollard, and P. Bianco, "Thrust balance characterization of a $200 \mathrm{~W}$ quad confinement thruster for high thrust regimes," IEEE Transactions on Plasma Science, vol. 43, no. 1, pp. 185-189, 2015.

[4] D. M. Goebel, Y. Hirooka, and T. A. Sketchley, "Large-area lanthanum hexaboride electron emitter," Review of Scientific Instruments, vol. 56, no. 9, pp. 1717-1722, 1985.

[5] D. M. Goebel and R. M. Watkins, "Compact lanthanum hexaboride hollow cathode," Review of Scientific Instruments, vol. 81, no. 8, pp. 1-7, 2010.

[6] D. M. Goebel and R. M. Watkins, "LaB6 Hollow Cathodes for Ion and Hall Thrusters," in 41st AIAA/ASME/SAE/ASEE Joint Propulsion Conference and Exhibit, no. 10-13 July, (Tucson, Arizona), pp. 1-9, 2005.

[7] R. O. Jenkins, "A review of thermionic cathodes," Vacuum, vol. 19, no. 8, pp. 353-359, 1969.

[8] H. E. Gallagher, "Poisoning of LaB6 cathodes," Journal of Applied Physics, vol. 40, no. 1, pp. 44-51, 1969.

[9] C. Oshima and S. Kawai, "Auger electron spectroscopy study of oxidation on lanthanum hexaboride," Applied Physics Letters, vol. 23, no. 5, pp. 215-216, 1973.

[10] C. Oshima, E. Bannai, T. Tanaka, and S. Kawai, "Carbon layer on lanthanum hexaboride (100) surface," Japanese Journal of Applied Physics, vol. 16, no. 6, pp. 965-969, 1977.

[11] E. Oks, Plasma Cathode Electron Sources. Weinheim, Germany: WILEY-VCH Verlag GmbH \& Co. KGaA, 2006.

[12] S. Weis, K.-H. Schartner, H. Löb, and D. Feili, "Development of a capacitively coupled insert-free RF-neutralizer," in Proceedings of the International Electric Propulsion Conference 2005 (IEPC05), (Princeton), pp. IEPC-2005-086, 2005.

[13] S. Jahanbakhsh, M. Satir, and M. Celik, "Study of electron current extraction from a radio frequency plasma cathode designed as a neutralizer for ion source applications," Review of Scientific Instruments, vol. 87, no. 2, 2016.

[14] F. Scholze, M. Tartz, and H. Neumann, "Inductive coupled radio frequency plasma bridge neutralizer," Review of Scientific Instruments, vol. 79, no. 2, p. 02B724, 2008.

[15] K. D. Diamant, "Resonant Cavity Hollow Cathode," in 41st AIAA/ASME/SAE/ASEE Joint Propulsion Coference \& Exhibit, no. 1013 July, (Tucson, Arizona), pp. AIAA 2005-3662, 2005.

[16] N. Yamamoto, Y. Hiraoka, and H. Nakashima, "Neutralizer for Miniature Ion Engines," in 33rd International Electric Propulsion Conference, (Washington, D. C.), pp. 1-6, 2013.

[17] H. Kamhawi, J. E. Foster, and M. J. Patterson, "Operation of a Microwave Electron Cyclotron Resonance Cathode," in 40th AIAA/ASME/SAE/ASEE Joint Propulsion Conference \& Exhibit, no. 11 -14 July, (Fort Lauderdale, Florida), pp. AIAA 2004-3819, 2004.

[18] B. Longmier, S. Baalrud, and N. Hershkowitz, "Nonambipolar electron source," Review of Scientific Instruments, vol. 77, no. 11, p. 113504 2006.

[19] A. Gurciullo, A. K. Knoll, and P. Bianco, "Experimental Performance Characterization of a Novel Direct Current Cold Cathode Neutralizer for Electric Thruster Applications," in 52nd AIAA/SAE/ASEE Joint Propulsion Conference, (Salt Lake City, Utah), pp. AIAA 2016-4945, 2016.

[20] V. V. Zhurin, H. R. Kaufman, and R. S. Robinson, "Physics of closed drift thrusters," Plasma Sources Science and Technology, vol. 8, no. 1, pp. R1-R20, 1999.

[21] V. Khayms, Advanced Propulsion for Microsatellites. Doctor of philosophy in aeronautics \& astronautics, Massachusetts Institute of Technology, 2000.

[22] D. P. Schmidt, N. B. Meezan, W. A. H. Jr, and M. A. Cappelli, "A lowpower, linear-geometry Hall plasma source with an open electron-drift," Plasma Sources Science and Technology, vol. 9, no. 1, p. 68, 2000.

[23] FEMM website, http://www.femm.info Last accessed 03 July 2017.

[24] E. M. Oks, "Physics and techniques of plasma electron sources," Plasma Sources Science and Technology, vol. 1, pp. 249-255, 1992.

[25] D. M. Goebel and I. Katz, Fundamentals of Electric Propulsion - Ion and Hall Thrusters. Hoboken, New Jersey: John Wiley and Sons, 2008.

[26] H. Watanabe, T. Deguchi, C. Ota, J. Sato, S. Takeda, Y. Miura, Y. Sato, M. Ichimura, and H. Takegahara, "Performance Evaluation of Radio Frequency Plasma Cathode for Hall Effect Thruster," in 34th International Electric Propulsion Conference, (Hyogo-Kobe, Japan), pp. 1-8, 2015.
[27] S. Jahanbakhsh and M. Celik, "Experimental Study of the Effects of Different Design Parameters on the Plasma Characteristics and the Extracted Current of a Prototype Radio-Frequency Plasma Cathode," in 34th International Electric Propulsion Conference, (Hyogo-Kobe), pp. IEPC-2015-108, 2015.

[28] K. D. Diamant, "Resonant Cavity Hollow Cathode Progress," in 29th International Electric Propulsion Conference, (Princeton), 2005.

[29] M. C. Edgar and S. G. Bilén, "Design and Testing of a High Power Electron Cyclotron Resonance Neutralizer," in 43rd AIAA/ASME/SAE/ASEE Joint Propulson Conference \& Exhibit, no. 8 -11 July, (Cincinnati), pp. AIAA 2007-5289, 2007.

[30] B. W. Longmier and N. Hershkowitz, "Electrodeless Plasma Cathode for Neutralization of Ion Thrusters," in 41st AIAA/ASME/SAE/ASEE Joint Propulsion Coference \& Exhibit, no. 10-13 July, (Tucson, Arizona), pp. AIAA 2005-3856, 2005.

[31] B. Longmier and N. Hershkowitz, "Improved operation of the nonambipolar electron source," Review of Scientific Instruments, vol. 79, no. 9, 2008.

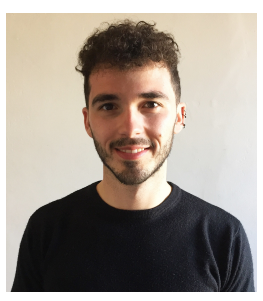

Antonio Gurciullo received the BSc degree in Aerospace Engineering from Politecnico di Torino, Torino, Italy, in 2013 and a MSc degree in Electrophysics from the Royal Institute of Technology (KTH), Stockholm, Sweden, in 2015. He is currently pursuing the $\mathrm{PhD}$ degree in Electronic Engineering at University of Surrey, Guildford, United Kingdom. $\mathrm{He}$ is a member of the Surrey Plasma Dynamics and Propulsion Group at Surrey Space Centre and his research interest includes the development of alternative neutralizers for plasma propulsion applications which operate at low temperature, low power and with molecular propellants, such as water vapour, air and carbonaceous compounds.

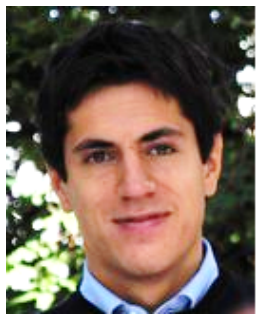

Andrea Lucca Fabris received the BSc and MSc degrees in Aerospace Engineering from the University of Padova, Padova, Italy, in 2007 and 2009, and the $\mathrm{PhD}$ in science technology and measurement for space from the same institution in 2014. From 2014 to 2015 he was a postdoctoral research fellow with Stanford University, Stanford, CA, USA and on 2016 he joined the Surrey Space Centre, Guildford, UK. His research interests include the experimental characterisation and numerical simulation of advanced plasma sources for space propulsion applications.

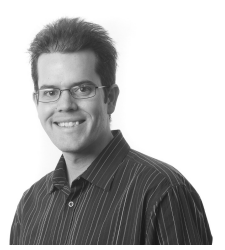

Aaron Knoll received his Bachelors of Aerospace Engineering (2003) and a Masters of Applied Science in Aerospace (2005) from Carleton University in Ottawa, Canada. He received his $\mathrm{PhD}$ (2010) from Stanford University, where he was involved with the research of instability driven electron transport within Hall Effect Thrusters. Aaron joined the Surrey Space Centre in 2010, and led the Surrey Plasma Dynamics and Propulsion Group from 2012 to 2017. $\mathrm{He}$ is currently a Senior Lecturer in Spacecraft Engineering at Imperial College London, with a research focus on the development of novel low power plasma propulsion technologies for small spacecraft. 